\title{
Rate of temporal discounting decreases with amount of reward
}

\author{
LEONARD GREEN, JOEL MYERSON, and EDWARD MCFADDEN \\ Washington University, St. Louis, Missouri
}

\begin{abstract}
The present, subjective value of a delayed reward is a decreasing function of the duration of the delay. This phenomenon is termed temporal discounting. To determine whether the amount of the reward influences the rate of temporal discounting, we had subjects choose between immediate and delayed hypothetical rewards of different amounts $(\$ 100, \$ 2,000, \$ 25,000$, and $\$ 100,000$ delayed rewards). As predicted by psychological models of the choice process, hyperbolic functions described the decrease in the subjective value of the delayed reward as the time until its receipt was increased $\left(R^{2} \mathrm{~s}\right.$ from .86 to .99$)$. Moreover, hyperbolic functions consistently provided more accurate descriptions of the data than did exponential functions predicted by an economic model of discounted utility. Rate of discounting decreased in a negatively accelerated fashion as the amount of the delayed reward increased, leveling off by approximately $\$ 25,000$. These findings are interpreted in the context of different psychological models of choice, and implications for procedures to enhance selfcontrol are discussed.
\end{abstract}

Temporal discounting refers to the fact that the greater the delay to a future reward, the less its present, subjective value. For example, most people would prefer $\$ 1,000$ now over the same amount in 6 months. This is presumably because the subjective value of the delayed $\$ 1,000$ is less; that is, people discount its value. Although $\$ 1,000$ now would obviously be preferable to $\$ 800$ now, people may discount delayed rewards to such a degree that they might well prefer $\$ 800$ now to the $\$ 1,000$ in 6 months.

Temporal discounting has been used to explain the preference reversal phenomenon. Although an individual may choose $\$ 800$ now to $\$ 1,000$ in 6 months, the same individual might well reverse his or her preference when an equal delay is added prior to the receipt of the amounts. That is, were a year to be added to the delay before the receipt of the amounts, the same individual might now prefer the $\$ 1,000$ in one and a half years to the $\$ 800$ in one year. Such reversals pose problems for the standard economic model of intertemporal choice (Koopmans, 1960; Samuelson, 1937) because they violate the stationarity assumption of discounted utility theory (Strotz, 1956; for a review, see Loewenstein, 1992). According to this assumption, preference should remain invariant when the delays to both amounts are increased or decreased equally.

The preference-reversal phenomenon is important for an understanding of impulsivity and self-control. Impulsivity can be defined as the choice of a smaller, more immediate reward over a larger, but more delayed reward;

A preliminary report of these findings was presented at the 1994 meeting of the Psychonomic Society in St. Louis. E. McFadden is now at the University of Connecticut. Correspondence concerning this manuscript should be addressed to L. Green or J. Myerson, Department of Psychology, Washington University, Campus Box 1125 , St. Louis, MO 63130 (e-mail: Igreen@artsci.wustl.edu). self-control would be exemplified by the choice of the larger, more delayed reward over the smaller, less delayed reward (e.g., Green, 1982; Logue, 1988; Rachlin, 1974; Rachlin \& Green, 1972). The occurrence of preference reversals (in which the smaller reward is preferred when the delays to both rewards are brief but the larger reward is preferred when the delays to both rewards are long) suggests that impulsivity may be a characteristic of the choice situation as much as a characteristic of the individual making the choice. That is, the same individual who acts impulsively when the smaller reward is available immediately may nevertheless show self-control and choose the larger reward when both rewards are delayed, even though the larger reward is more delayed than the smaller.

The form of the function describing the change in subjective value over time is critical in explaining the preference-reversal phenomenon and related aspects of self-control. Two forms of temporal discounting functions have been proposed, an exponential model and a hyperbolic model (e.g., Ainslie, 1975, 1992; Loewenstein, 1992; Mazur, 1987; Samuelson, 1937), along with variations on these forms (Green, Fry, \& Myerson, 1994; Loewenstein \& Prelec, 1992; Myerson \& Green, 1995). The exponential model posits that people make their decisions about the value of future rewards on the basis of the assumption of a constant hazard rate (i.e., people assume that the probability that something will happen to prevent receipt of a reward does not change over time). Conceptualized in the same terms, the hyperbolic model implies that the hazard rate decreases over time. Thus, the two models imply alternative representations of the risks involved in waiting for future outcomes (Green \& Myerson, 1996).

The exponential function, favored by economists, is given by the following equation: 


$$
V(A)=A e^{-k_{A} D},
$$

where $V(A)$ is the subjective value of a future reward of amount $A, D$ is the delay to its receipt, and $k_{\mathrm{A}}$ represents the parameter describing the rate of decrease in its value. The hyperbolic function, favored by psychologists, takes the following form:

$$
V(A)=A /\left(1+k_{\mathrm{A}} D\right)
$$

Fit to the same data, the hyperbola shows an initially steeper decrease in subjective value than the exponential but a more gradual decrease in value as the delay increases, reflecting the different risk assumptions of the two models.

If it is assumed that the rate of temporal discounting (indexed by the parameter $k_{\mathrm{A}}$ ) is independent of the amount of the reward, then the exponential model (Equation 1) leads to the prediction that the relative value of two delayed rewards will remain the same if the delays to both rewards are increased or decreased equally. Thus, according to the exponential model, the preferencereversal phenomenon should not occur. In contrast, given the same assumption (i.e., $k_{\mathrm{A}}$ is independent of amount), the hyperbolic model (Equation 2) predicts that preference may reverse with equal changes in the delays to both rewards (Ainslie, 1992; Mazur, 1987).

Recent findings suggest that larger rewards are discounted less steeply than smaller rewards (Benzion, Rapoport, \& Yagil, 1989; Myerson \& Green, 1995; Raineri \& Rachlin, 1993; Thaler, 1981). However, these studies have examined either few amounts or delays, or a narrow range of amounts or delays, or have compared discounting rates using different groups of participants at the different amounts. If the amount dependency of discounting rate is confirmed, this will have important theoretical consequences. As Green and Myerson (1993) have shown, if the observed amount dependency is incorporated into the models so that $k_{\mathrm{A}}$ decreases with amount of reward, then both models, the exponential as well as the hyperbolic, predict the preference-reversal phenomenon (see Green \& Myerson, 1993, for a fuller discussion of this issue).

Given the importance of this issue, with its fundamental implications for models of decision making, it becomes necessary to rigorously test the hypothesis of amount-dependent discounting suggested by previous research. Moreover, if the decrease in discounting rate with increased amount is validated, then the details of the relationship between discounting rate and amount need careful examination. Thus, an important question becomes whether there is a point beyond which further increases in amount have no additional effect on discounting rate, and if so, where that point is located. No published data address this question.

Therefore, the present study was designed to investigate more fully the effect of amount of reward on temporal discounting. Specifically, subjects were exposed to choices involving four different delayed amounts over a wide range of delays in order to evaluate the relationship between amount and discounting rate. In addition, although current psychological evidence favors a hyperbolic model, most economic theorizing remains based on the exponential model. Consequently, the present study also compared the fit of the hyperbolic and exponential models at each amount. By evaluating the exponential and hyperbolic models at a number of delayed amounts, the present study is intended to provide a more complete and rigorous assessment of the two models.

\section{METHOD}

\section{Subjects}

Twenty-four Washington University undergraduate students (20 women and $4 \mathrm{men}$ ) between the ages of 18 and 23 years $(M=20.6$ years) were paid for their voluntary participation in this study.

\section{Procedure}

The subjects were tested individually for about $50 \mathrm{~min}$ in a quiet room containing a table and two chairs. The procedure, similar to that used in Green et al. (1994), required participants to make a series of choices regarding hypothetical amounts of money, one available immediately and one available after a specified delay. The delayed and immediate amounts were presented on $4 \times 6 \mathrm{in}$. index cards. The subjects made a series of choices between a fixedamount reward (e.g., \$100) that could be obtained after a delay (e.g., 3 months) and an immediately obtainable reward that varied in amount (e.g., from $\$ 1$ to $\$ 99$ ). For example, the subject was given a choice between $\$ 100$ in 3 months or $\$ 85$ now. The subject indicated his/her preference by pointing to one of the two cards (either the delayed, fixed-amount reward or the immediate reward).

The subjects were read the following instructions prior to testing:

The purpose of the present study is to compare your preference between different amounts of money available at different points in time. In this study you will be asked to make a series of choices between hypothetical monetary alternatives. As you can see, there are two sets of cards in front of you. The cards on your left will offer you an amount of money to be paid right now. This amount will vary from card to card. During this study, the amount on the cards to your right will be either $\$ 100, \$ 2,000, \$ 25,000$, or $\$ 100,000$, but its payment will be delayed.

Please look at the example cards at this time. It will be your job to choose between the two cards presented and to point to the card you would prefer. It is important to base your decision on only the two cards presented on each trial. Do not base your decision on sets of cards previously seen or ones you expect to see. There are no correct or incorrect choices. We are interested in the option you would prefer.

You will be given five practice trials before you begin, and I will turn the cards for you. You will get plenty of practice so do not worry if you feel that you do not understand completely at this time. During the practice please ask me as many questions as you like because once practice is over I can no longer answer your questions.

The seven delays used at each of the four fixed-amount rewards (i.e., $\$ 100, \$ 2,000, \$ 25,000$, and $\$ 100,000$ ) were 3 months, 6 months, 1 year, 3 years, 5 years, 10 years, and 20 years. The immediate-reward cards depicted 24 values ranging from $1 \%$ to $99 \%$ of the delayed fixed-amount reward to which they were compared (e.g., when the fixed amount was $\$ 100$, the immediate rewards were $\$ 1, \$ 2.50, \$ 5, \$ 7.50, \$ 10, \$ 15, \$ 20, \$ 25, \$ 30, \$ 35, \$ 40$, $\$ 45, \$ 50, \$ 60, \$ 65, \$ 70, \$ 75, \$ 80, \$ 85, \$ 90, \$ 92.50, \$ 95, \$ 97.50$, and $\$ 99$ ). For each fixed amount at each delay, the immediate rewards were presented in either ascending or descending order. When immediate rewards were presented in ascending order, the amount of the immediate reward judged equal in subjective value to the delayed reward was calculated as the average of the first immediate amount preferred over the delayed reward and the preced- 
ing immediate amount. When immediate rewards were presented in descending order, the amount of the immediate reward judged equal in subjective value to the delayed reward was calculated as the average of the last immediate amount preferred over the delayed reward and the next immediate amount.

The orders of presentation of the immediate amount and the delayed amounts were both counterbalanced. Half of the participants were offered the fixed-amount, delayed rewards from $\$ 100$ up to $\$ 100,000$; the other half were offered the fixed-amount rewards in the reverse order. For the briefest delay at each fixed amount, half of the subjects were given the immediate amounts in ascending order and half in descending order; at the next delay for this fixed amount, the half that had just been given the immediate amounts in ascending order now received them in descending order, and vice versa. This alternation continued until all seven delays had been tested at a given fixed amount, at which point the next fixedamount condition began and the immediate rewards were presented in the opposite order to that at the last fixed amount.

\section{RESULTS}

Table 1 gives the amount of the immediate reward judged equal in subjective value to the delayed reward, expressed as a proportion of the amount of the delayed reward. Data are group medians. The median was used because the distributions of subjective equivalence points are usually negatively skewed when delays are brief and positively skewed when delays are long (Rachlin, Raineri, $\&$ Cross, 1991). The skew is a consequence of the limits imposed on subjects' choices; that is, the subjective value of a delayed reward is less than its nominal value and greater than zero.

Two results are apparent from Table 1. First, temporal discounting occurred at each of the four amounts: Reading down the columns, one sees that the amount of the immediate reward (expressed as a proportion of the delayed amount) that is subjectively equivalent to the delayed reward decreases systematically with delay for each of the four delayed amounts. Second, the degree of discounting decreased as the amount of the delayed reward increased: Reading across the rows, one sees that the amount of the immediate reward that is subjectively equivalent to the delayed reward (expressed as a proportion of the delayed amount) increases systematically from $\$ 100$ to $\$ 25,000$, with no further change between $\$ 25,000$ to $\$ 100,000$.

Temporal discounting functions (e.g., Equations 1 and 2) attempt to describe decreases in subjective value such

Table 1

Amount of the Immediate Reward Judged Equal in Subjective Value to the Delayed Reward, Expressed as a Proportion of the Amount of the Delayed Reward

\begin{tabular}{lcccc}
\hline & \multicolumn{3}{c}{ Amount } \\
\cline { 2 - 5 } \multicolumn{1}{c}{ Delay } & $\$ 100$ & $\$ 2,000$ & $\$ 25,000$ & $\$ 100,000$ \\
\hline 3 months & .83 & .95 & .97 & .98 \\
6 months & .78 & .91 & .95 & .95 \\
1 year & .68 & .89 & .91 & .93 \\
3 years & .60 & .73 & .78 & .73 \\
5 years & .40 & .55 & .68 & .65 \\
10 years & .35 & .38 & .58 & .50 \\
20 years & .20 & .23 & .30 & .30 \\
\hline
\end{tabular}

as one sees reading down the columns in Table 1. Figure 1 shows temporal discounting functions for the four delayed-reward amounts. For each delay, the data point represents the group median of the immediate rewards judged equal in value to the delayed reward. The best fitting exponential (Equation 1) and hyperbolic (Equation 2) functions are represented by dashed and solid curves, respectively. Also shown is the proportion of variance accounted for $\left(R^{2}\right)$ by each equation. For each of the four amounts, the proportion of variance accounted for by the hyperbolic was larger than that accounted for by the exponential function. This was because, as may be seen in the figure, the exponential typically overestimated the subjective value of the delayed reward at briefer delays, and underestimated the subjective value at longer delays.

For both exponential and hyperbolic functions, the value of the discounting parameter $k$ decreased with the amount of the delayed reward, leveling off by approximately $\$ 25,000$. Focusing on the hyperbolic model, which provided the better fit to the data, the same pattern was also observed when the hyperbolic function was fit to each subject's data separately (see Table 2). This may be seen in Figure 2, which shows the $k$ value of the hyperbolic function fit to the median values and also the median of the individual $k$ values, both of which decrease as amount increases from $\$ 100$ to $\$ 25,000$. The pattern of decreases in $k$ with increases in amount for the group median subjective values was confirmed statistically using nonlinear regression to compare the discount parameters for larger and smaller amounts [ $\$ 100$ vs. $\$ 2,000$, $t(12)=2.88, p<.05 ; \$ 2,000$ vs. $\$ 25,000, t(12)=5.57$, $p<.01 ; \$ 25,000$ vs. $\$ 100,000, t(12)=1.91$, n.s.]. ${ }^{1}$

It may be recalled that for each combination of the seven delays and the four delayed amounts, half of the subjects were studied with the immediate rewards presented in order of increasing amount until preference reversed and half were studied with the immediate rewards presented in order of decreasing amount until preference reversed. In addition to analyzing the data by combining the subjectively equivalent values from the ascending and descending series, as shown in Figure 1, we also analyzed the data by taking the median subjectively equivalent values from the ascending and descending series separately. As may be seen in Figure 3, there were no systematic differences between the ascending and descending series with respect to the amounts of the immediate reward judged subjectively equivalent to the delayed rewards. This was confirmed statistically by nonlinear regression, which revealed no significant differences between the discount parameters for ascending and descending series at any of the four delayed amounts $[\$ 100, t(12)<$ $1.0 ; \$ 2,000, t(12)<1.0 ; \$ 25,000, t(12)=2.03 ; \$ 100,000$, $t(12)<1.0]$.

Figure 4 presents data from 4 individual subjects (the $1 \mathrm{st}, 8 \mathrm{th}, 16 \mathrm{th}$, and 24 th subjects studied). For each subject at each delayed amount, the subjective value of the delayed reward at each of the seven delays is shown, along with the best fitting hyperbolic function (Equa- 

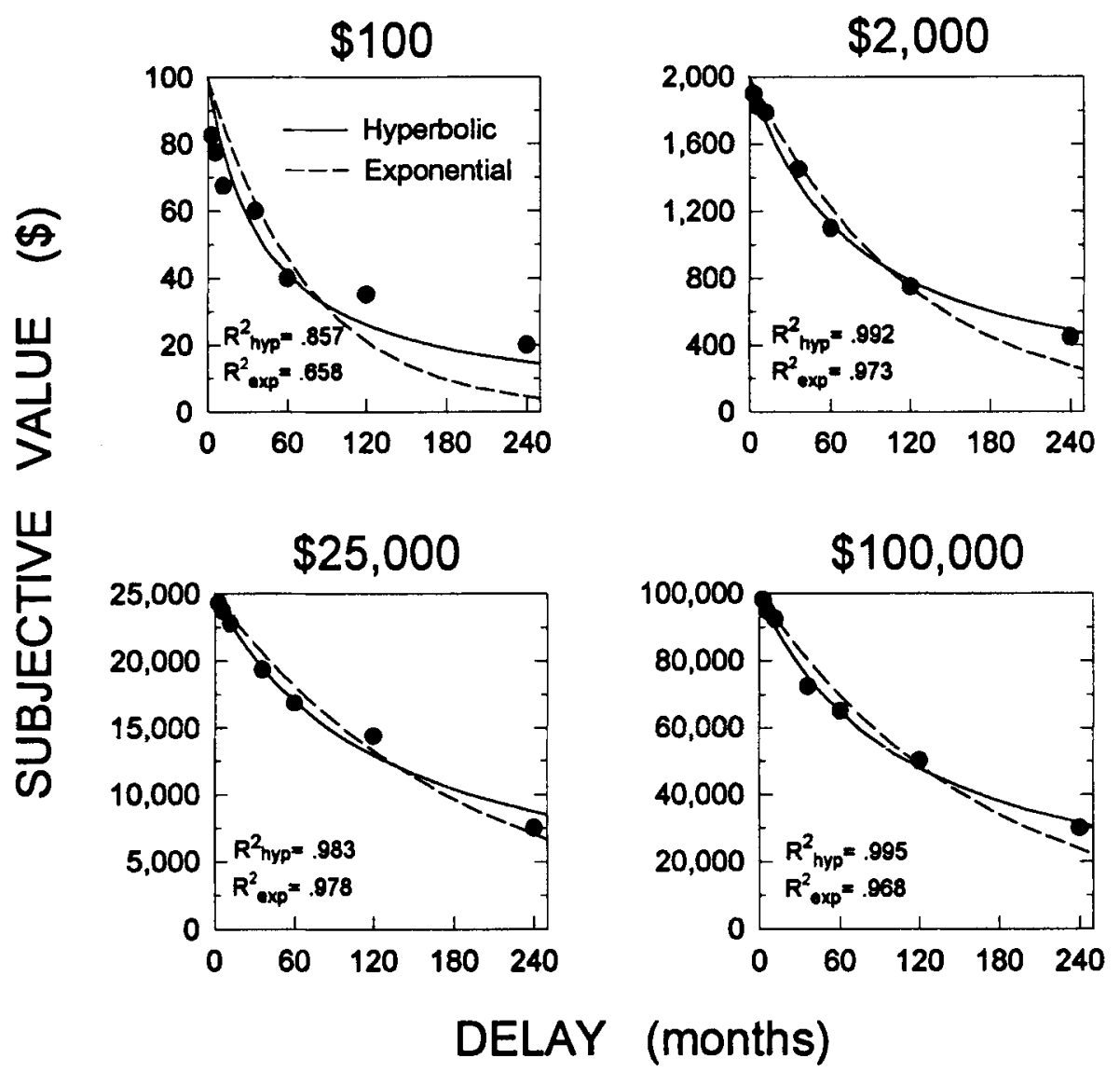

Figure 1. Present, subjective value of the delayed reward as a function of the time until its receipt. Data for the four delayed amounts are shown in separate panels. Data points represent the median amount of immediate reward judged equal in subjective value to the delayed reward. Solid and dashed curves represent the best fitting hyperbolic and exponential discounting functions, respectively.

tion 2). Although there is some variability, the same pattern of decreases in discounting rate as amount increases observed at the group level typically holds at the individual level as well.

\section{DISCUSSION}

The present findings demonstrate that the rate at which the value of a future reward is discounted depends on the amount of the reward. More specifically, the rate of discounting decreased in a negatively accelerated fashion as the amount of the reward was increased from $\$ 100$ to $\$ 25,000$. Increasing the amount from $\$ 25,000$ to $\$ 100,000$ produced no further decrease in discounting rate. Given amount-dependent discounting, the exponential model (like the hyperbolic model) predicts that preference between smaller, sooner rewards and larger, later rewards will depend on the time at which a choice is made. Thus, preference reversals in and of themselves cannot be taken as evidence for the hyperbolic and against the exponential model. Nevertheless, the present results clearly show that a hyperbola provides a better description of the relation between subjective value and delay than does an exponential decay function across a wide range of amounts (from $\$ 100$ to $\$ 100,000$ ). In fact, the hyperbolic model accounted for more than $98 \%$ of the variance at the three largest amounts.

Data from studies using a variety of different procedures with real and hypothetical outcomes in human and animal subjects converge to show that the nature of the processes involved in decisions about immediate and delayed rewards is well captured by a hyperbolic discounting model. Although the present results were obtained asking subjects about their choices between hypothetical rewards, there are good reasons to believe that choices regarding hypothetical rewards are reflective of the processes involved in choosing between real rewards. It has been shown that when animals choose between delayed food rewards, preference decreases hyperbolically with delay (Chung \& Herrnstein, 1967). With humans, Kirby and Marakovic (1995) have shown that a hyperbolic model described discounting of small, real monetary rewards better than an exponential model.

The present findings confirm that the rate of discounting, as measured by the $k$ parameter in the hyperbolic model, decreases with the amount of a delayed reward. 
Table 2

Values of $k$ (the Discount Rate Parameter) and $\boldsymbol{R}^{\mathbf{2}}$ for Fits of the Hyperbolic Model (Equation 2) to Individual Subjects' Data

\begin{tabular}{|c|c|c|c|c|c|c|c|c|}
\hline \multirow[b]{3}{*}{ Subject } & \multicolumn{8}{|c|}{ Amount } \\
\hline & \multicolumn{2}{|c|}{$\$ 100$} & \multicolumn{2}{|c|}{$\$ 2,000$} & \multicolumn{2}{|c|}{$\$ 25,000$} & \multicolumn{2}{|c|}{$\$ 100,000$} \\
\hline & $k$ & $R^{2}$ & $k$ & $R^{2}$ & $k$ & $R^{2}$ & $k$ & $R^{2}$ \\
\hline 1 & 0.216 & 0.592 & 0.038 & 0.843 & 0.010 & 0.800 & 0.006 & 0.832 \\
\hline 2 & 0.764 & 0.966 & 0.119 & 0.852 & 0.057 & 0.888 & 0.056 & 0.819 \\
\hline 3 & 0.015 & 0.471 & 0.010 & 0.847 & 0.004 & 0.491 & 0.001 & 0.955 \\
\hline 4 & - & 0.000 & - & 0.000 & 0.497 & 0.809 & - & 0.000 \\
\hline 5 & 0.161 & 0.919 & 0.137 & 0.684 & 0.042 & 0.930 & 0.021 & 0.743 \\
\hline 6 & 0.041 & 0.637 & 0.018 & 0.350 & 0.011 & 0.675 & 0.008 & 0.675 \\
\hline 7 & 0.054 & 0.157 & -- & 0.000 & - & 0.000 & - & 0.000 \\
\hline 8 & 0.025 & 0.898 & 0.028 & 0.919 & 0.007 & 0.935 & 0.008 & 0.874 \\
\hline 9 & 0.019 & 0.905 & 0.006 & 0.780 & 0.004 & 0.664 & 0.003 & 0.590 \\
\hline 10 & 0.028 & 0.000 & 0.009 & 0.685 & 0.003 & 0.371 & 0.002 & 0.735 \\
\hline 11 & 0.023 & 0.965 & 0.015 & 0.919 & 0.008 & 0.916 & 0.005 & 0.990 \\
\hline 12 & 0.012 & 0.064 & 0.012 & 0.913 & 0.010 & 0.940 & 0.009 & 0.995 \\
\hline 13 & 0.006 & 0.190 & 0.015 & 0.966 & 0.011 & 0.928 & 0.009 & 0.990 \\
\hline 14 & - & 0.000 & 0.002 & 0.497 & 0.001 & 0.855 & 0.001 & 0.835 \\
\hline 15 & 0.010 & 0.941 & 0.012 & 0.900 & 0.006 & 0.913 & 0.008 & 0.868 \\
\hline 16 & 0.093 & 0.861 & 0.008 & 0.950 & 0.002 & 0.655 & 0.025 & 0.713 \\
\hline 17 & 0.063 & 0.921 & 0.012 & 0.890 & 0.008 & 0.840 & 0.006 & 0.966 \\
\hline 18 & 0.011 & 0.960 & 0.012 & 0.922 & 0.012 & 0.980 & 0.014 & 0.982 \\
\hline 19 & 0.007 & 0.891 & 0.006 & 0.830 & 0.003 & 0.430 & - & 0.000 \\
\hline 20 & - & 0.000 & 0.001 & 0.041 & 0.001 & 0.621 & 0.005 & 0.332 \\
\hline 21 & 0.198 & 0.910 & 0.014 & 0.990 & 0.010 & 0.916 & 0.008 & 0.962 \\
\hline 22 & 0.007 & 0.729 & 0.139 & 0.623 & - & 0.000 & - & 0.000 \\
\hline 23 & 0.014 & 0.915 & 0.015 & 0.571 & - & 0.000 & - & 0.000 \\
\hline 24 & 0.029 & 0.425 & 0.005 & 0.945 & 0.004 & 0.864 & 0.003 & 0.987 \\
\hline
\end{tabular}

Note-Dashes indicate that the value of $k$ is omitted because the poor fit made the parameter estimate meaningless; $R^{2}$ of 0.000 indicates that the function accounted for less of the variance than did the mean.

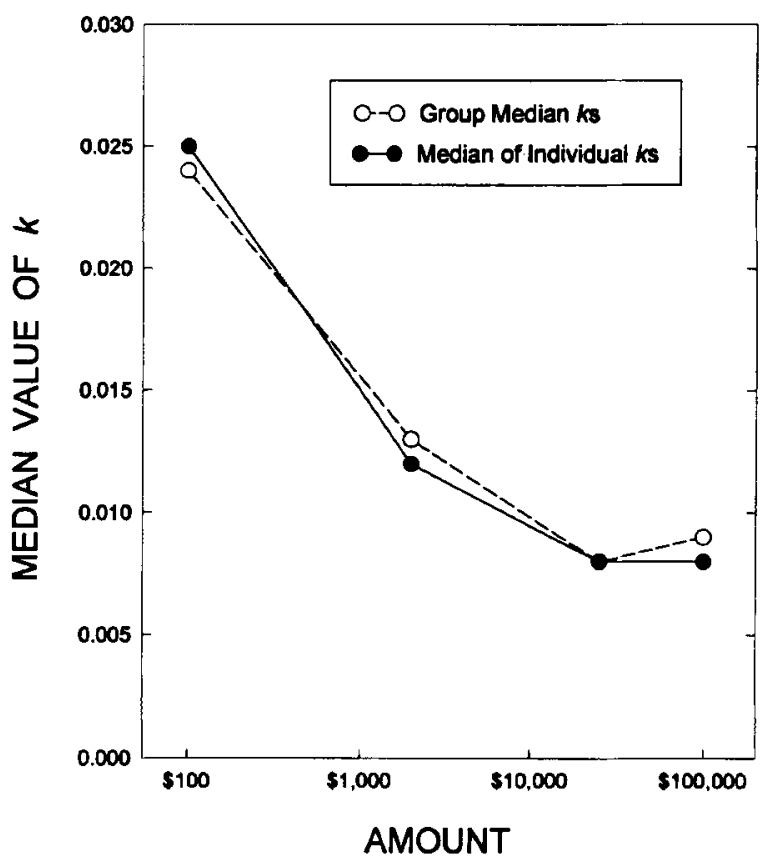

Figure 2. Rate of discounting $(k)$ as a function of the amount of the delayed reward. Both the estimates of $k$ based on fits of the hyperbolic model to the group median subjective values and the median of the estimates based on fits to the data from individual subjects are shown.
Although Kirby and Marakovic (1995) failed to find an amount effect, this discrepancy is likely due to the small range of the amounts studied ( $\$ 14.75$ to $\$ 28.50)$, as another study by these authors (Kirby \& Marakovic, 1996), which used a wider range ( $\$ 30$ to $\$ 85$ ), did find a decrease in discounting rate with increases in amount. In addition, the present results demonstrate that the rate of discounting levels off somewhere in the neighborhood of $\$ 25,000$. Raineri and Rachlin (1993) reported a decrease in discounting rate with three delayed amounts $(\$ 100$, $\$ 10,000$, and $\$ 1$ million) but failed to observe such a leveling off. However, their results are not inconsistent with ours. Rather, taken together with the present results, they aid in localizing the point at which the rate of discounting levels off (i.e., between $\$ 10,000$ and $\$ 25,000$ ).

Various explanations have been offered to account for the fact that rate of discounting decreases as the amount of the delayed reward increases. For example, it has been proposed that people maintain two different mental accounts for large and small amounts, analogous to a "savings" account and a "windfall" account (Loewenstein \& Thaler, 1989). This metaphor may have an intuitive appeal. However, at least three different mental accounts would be required to explain the three, significantly different discounting rates that were observed in the current study. If, as seems likely, even smaller values (e.g., under $\$ 10$ ) are discounted yet more steeply, then it would seem 

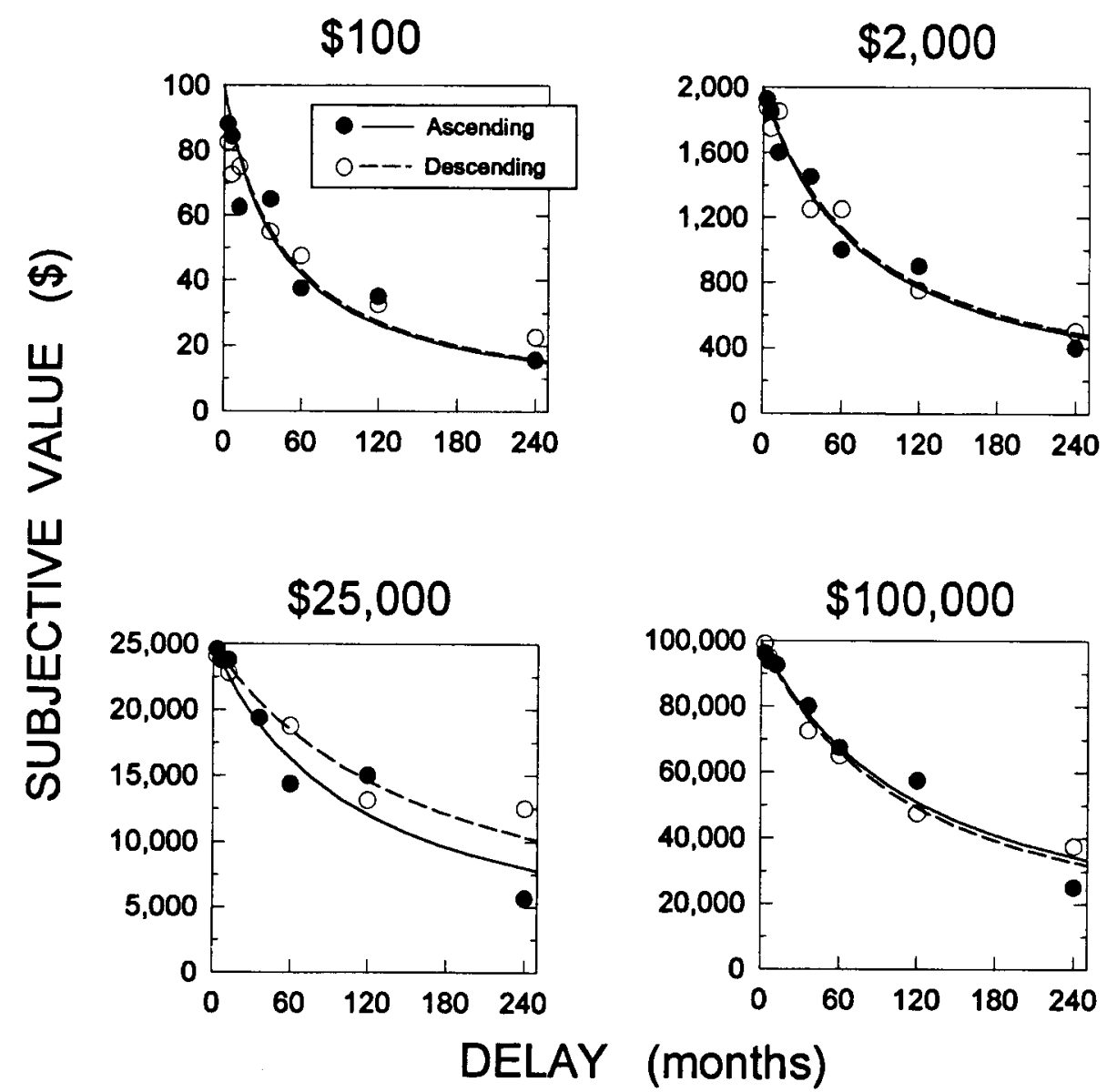

\begin{abstract}
Figure 3. Present, subjective value of the delayed reward as a function of the time until its receipt. Data for the four delayed amounts are shown in separate panels. Solid and dashed curves represent hyperbolic discounting functions fit to the median subjective values for the ascending and descending series, respectively.
\end{abstract}

more parsimonious to postulate a continuous relation between amount and discounting rate rather than four, or even more, discrete mental accounts.

Another approach to the problem of amount-dependent discounting has been suggested by Raineri and Rachlin (1993) as part of an effort to deal with the roles of consumption rate and duration in decision making. They assume that consumption takes time and that larger rewards take longer to consume than smaller rewards. They assume further that consumption rate increases with amount in a negatively accelerated manner. For example, a $\$ 10,000$ reward might be spent at a faster rate than a $\$ 100$ reward but not 100 times faster. In the context of their model, these assumptions give rise to an interaction between amount and delay, such that larger rewards are discounted at a lower rate.

Myerson and Green (1995) have suggested two possible explanations for the effect of amount on discounting rate. According to one hypothesis, noted in the introduction, hyperbolic discounting occurs because decisions are based on the implicit assumption that the likelihood that something will happen to prevent receipt of a future reward (i.e., the hazard rate) decreases over the waiting period. We have termed this hypothesis the expectedvalue model. From the perspective of the expected-value model, the phenomenon of amount-dependent discounting implies that larger rewards are associated with lower hazard rates than smaller rewards.

Hyperbolic discounting occurs in both animals and humans, suggesting a common evolutionary heritage, and it may be useful to consider the expected-value model in this context. That is, amount-dependent discounting may represent an adaptation to differences in the risks involved in foraging for larger and smaller rewards. These risks may be either ecological or cognitive (Green \& Myerson, 1996). For example, for a foraging animal, the ecological risk associated with waiting for a larger reward may be lower because, even if a competitor gets to it first, it is more likely that some of the reward will be left, whereas with a smaller reward, it is less likely that any would remain should a competitor find it. The cognitive risk reflects the fact that waiting for a reward involves 


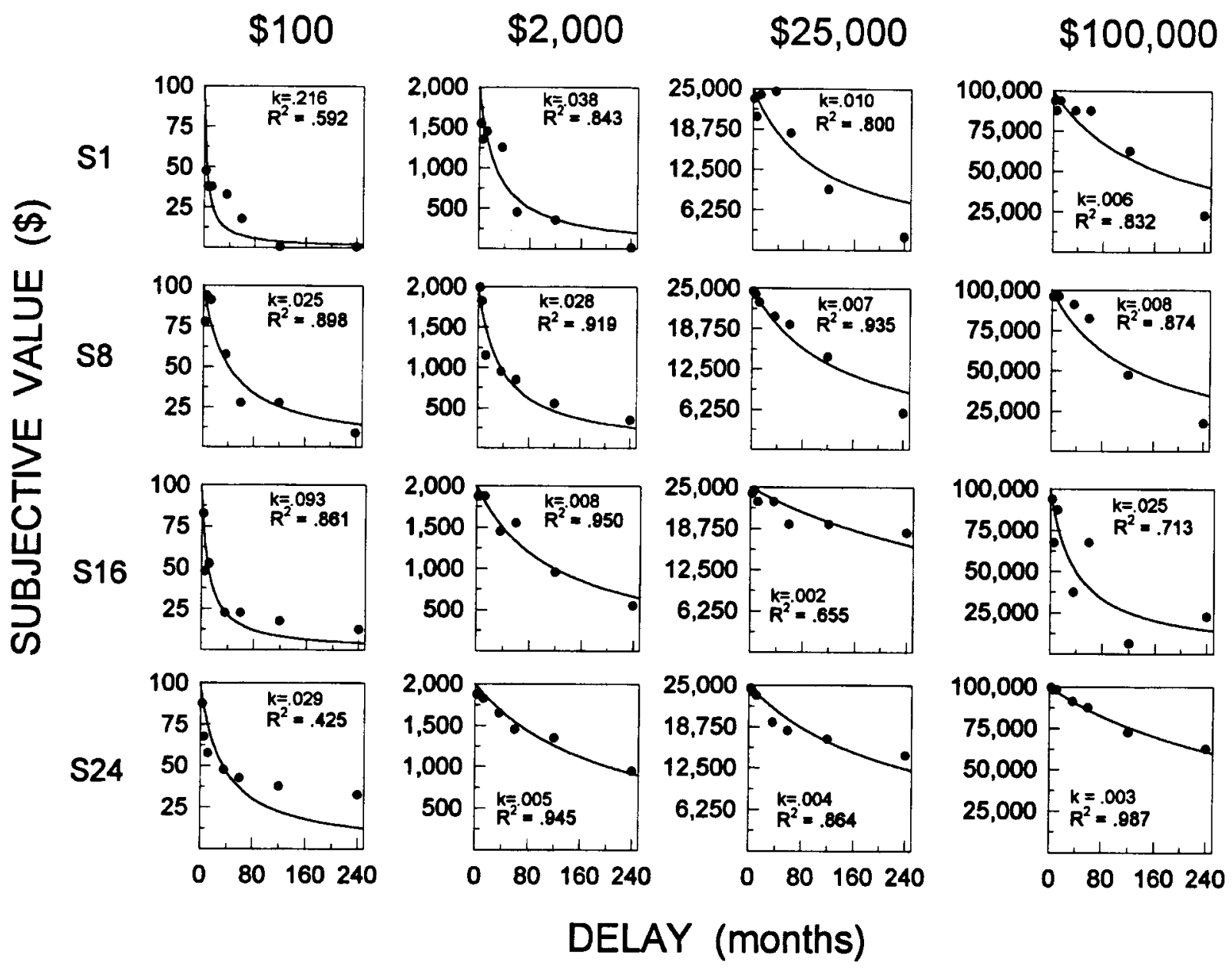

Figure 4. Present, subjective value of the delayed reward as a function of the time until its receipt. Data from four individual participants at each of the four delayed amounts are shown in separate panels. Solid curves represent the best fitting hyperbolic discounting function; the estimated discounting rate $(k)$ and the proportion of explained variance $\left(R^{2}\right)$ are given for each curve.

the possibility of forgetting when and where it may be obtained, and large rewards may be more salient, that is, more memorable.

According to another hypothesis, which we have termed the repeated-choice model, individuals make an implicit assumption regarding how often they are likely to have an opportunity to choose between a particular pair of alternatives, and the discounting rate is determined by the time between choice opportunities (Myerson \& Green, 1995). Larger amounts are discounted less steeply because individuals assume that choice opportunities involving such amounts are rarer than choice opportunities involving smaller amounts. Both models appear to be more easily testable than metaphorical hypotheses such as separate mental accounts. For example, the expected-value model might be evaluated by manipulating risks (e.g., by increasing salience while holding amount constant) and the repeated-choice model might be evaluated by explicitly varying the rate at which choice opportunities are presented (see Rachlin, Logue, Gibbon, \& Frankel, 1986, for a similar approach to testing a model of choice in- volving repeated gambles). Given both the accumulating evidence for the hyperbolic form of the discounting function and the present findings regarding the range and robustness of the effect of amount on discounting, future experiments should be aimed at distinguishing among the several theoretical explanations for amount-dependent hyperbolic discounting (e.g., mental accounts, consumption rates, expected value, and repeated choice).

Figure 5 illustrates how self-control depends on the difference between the rates at which large and small rewards are discounted. The heights of the bars represent the amounts of the two rewards; the solid curves represent discounting functions (i.e., subjective value as a function of time) for the large and small rewards, assuming that both are discounted at the same rate. The solid circle represents the crossover point: Before this point (i.e., at times to the left of the solid circle), the subjective value of the larger reward is greater than that of the smaller reward; after this point, the subjective value of the smaller reward is greater. Thus, if one chooses between the two rewards at time $T_{1}$, shortly before the smaller, more im- 


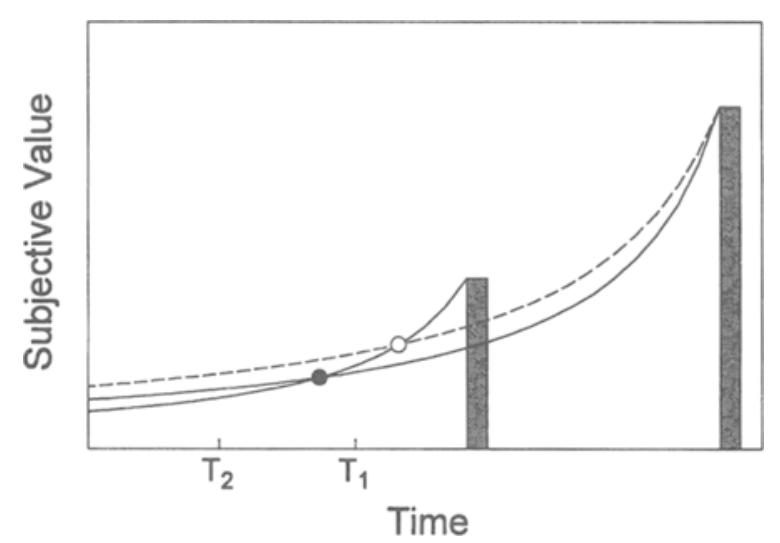

Figure 5. Subjective values of two delayed rewards as a function of the time at which the choice between them is made. The heights of the bars represent the amounts of the two rewards, the solid curves represent temporal discounting functions assuming that both rewards are discounted at the same rate, and the dashed curve represents the temporal discounting function for the larger reward assuming that it is discounted less steeply than the smaller reward. The circles represent points at which the two rewards are equivalent in subjective value, the solid circle under the assumption of amount-independent discounting and the open circle under the assumption of amountdependent discounting. $T_{1}$ and $T_{2}$ represent two choice points discussed in the text.

mediate reward could be received, the smaller reward will be preferred (i.e., it has the greater subjective value). However, if one chooses at time $T_{2}$, so that there is a longer interval until the smaller reward could be received, then the larger, more delayed reward will be preferred. The latter choice is often considered to reflect greater self-control relative to the more "impulsive" choice of the smaller, more immediate reward.

However, large and small rewards are not discounted at the same rate, as the present results demonstrate. The lower discounting rate associated with a larger reward is illustrated in Figure 5 by the dashed curve, and the resulting crossover point is represented by the open circle. Notice that when the discounting rate for the larger reward is less, the crossover point moves to the right, thus reducing the time interval over which "impulsive" behavior (i.e., the choice of the smaller over the larger reward) is observed. It follows that any manipulation that would further decrease the discounting rate for the larger reward would produce additional reductions in the likelihood of "impulsive" behavior.

Understanding the determinants of discounting rate and their role in the decision-making process is important in part because such understanding may suggest ways to enhance self-control. That is, different models suggest that different variables will influence choice between long-term and short-term interests, as represented by delayed, larger and more immediate, but smaller rewards, respectively. For example, the expected-value model implies that increasing confidence in the certainty of future rewards will decrease the rate at which they are discounted, thus increasing the likelihood that they will be chosen over smaller, more immediate rewards. Similarly, the repeated-choice model implies that increasing perception of the rarity of choice opportunities would also enhance self-control.

Finally, regardless of the theoretical mechanism underlying temporal discounting, increasing the perceived value of the delayed reward should result in greater selfcontrol, in the sense of extending the range of delays over which individuals will choose the larger, delayed reward over a more immediate but smaller reward. However, the present results show that although the rate of discounting decreases with amount of reward, it levels off at large amounts. This leveling off suggests that there will be limits to the effectiveness of efforts to increase self-control by increasing the subjective value of delayed rewards. Consequently, the effectiveness of a variety of approaches, including those suggested by the expectedvalue and repeated-choice models, needs to be explored.

\section{REFERENCES}

AINSLIE, G. (1975). Specious reward: A behavioral theory of impulsiveness and impulse control. Psychological Bulletin, 82, 463-496.

AINSLIE, G. (1992). Picoeconomics: The strategic interaction of successive motivational states within the person. Cambridge: Cambridge University Press.

Benzion, U., Rapoport, A., \& Yagil, J. (1989). Discount rates inferred from decisions: An experimental study. Management Science, 35, 270-284.

Chung, S.-H., \& Herrnstein, R. J. (1967). Choice and delay of reinforcement. Journal of the Experimental Analysis of Behavior, 10, 67. 74.

Gallant, A. R. (1987). Nonlinear statistical models. New York: Wiley GREEN, L. (1982). Self-control behavior in animals. In V. L. Smith (Ed.), Research in experimental economics (Vol. 2, pp. 129-150). Greenwich, CT: JAI Press.

Green, L., Fry, A. F., \& Myerson, J. (1994). Discounting of delayed rewards: A life-span comparison. Psychological Science, 5, 33-36.

Green, L., \& Myerson, J. (1993). Alternative frameworks for the analysis of self control. Behavior \& Philosophy, 21, 37-47.

Green, L., \& MYerson, J. (1996). Exponential versus hyperbolic discounting of delayed outcomes: Risk and waiting time. American Zoologist, 36, 496-505

Kirby, K. N., \& Marakovic, N. N. (1995). Modeling myopic decisions: Evidence for hyperbolic delay-discounting within subjects and amounts. Organizational Behavior \& Human Decision Processes, 64, 22-30.

Kirby, K. N., \& Marakovic, N. N. (1996). Delay-discounting probabilistic rewards: Rates decrease as amounts increase. Psychonomic Bulletin \& Review, 3, 100-104.

Koopmans, T. C. (1960). Stationary ordinal utility and impatience. Econometrica, 28, 287-309.

LOEWENSTEIN, G. (1992). The fall and rise of psychological explanations in the economics of intertemporal choice. In $\mathrm{G}$. Loewenstein \& J. Elster (Eds.), Choice over time (pp. 3-34). New York: Russell Sage

Loewenstein, G., \& Prelec, D. (1992). Anomalies in intertemporal choice: Evidence and an interpretation. Quarterly Journal of Economics, 107, 573-597.

LoEWENSTEIN, G., \& Thaler, R. H. (1989). Anomalies: Intertemporal choice. Journal of Economic Perspectives, 3, 181-193.

Logue, A. W. (1988). Research on self-control: An integrating framework. Behavioral \& Brain Sciences, 11, 665-679.

MaZur, J. E. (1987). An adjusting procedure for studying delayed reinforcement. In M. L. Commons, J. E. Mazur, J. A. Nevin, \& H. Rachlin (Eds.), Quantitative analyses of behavior: Vol. 5. The effect of delay and of intervening events on reinforcement value (pp. 55-73) Hillsdale, NJ: Erlbaum.

Myerson, J., \& Green, L. (1995). Discounting of delayed rewards: 
Models of individual choice. Journal of the Experimental Analysis of Behavior, 64, 263-276.

RaChlin, H. (1974). Self-control. Behaviorism, 2, 94-107.

Rachlin, H., \& Green, L. (1972). Commitment, choice and selfcontrol. Journal of the Experimental Analysis of Behavior, 17, $15-$ 22.

Rachlin, H., Logue, A. W., Gibbon, J., \& Frankel, M. (1986). Cognition and behavior in studies of choice. Psychological Review, 93, 33-45.

RaChlin, H., Raineri, A., \& Cross, D. (1991). Subjective probability and delay. Journal of the Experimental Analysis of Behavior, 55, 233-244.

RAINERI, A., \& RACHLIN, H. (1993). The effect of temporal constraints on the value of money and other commodities. Journal of Behavioral Decision Making, 6, 77-94.

SAMUELSON, P. A. (1937). A note on measurement of utility. Review of Economic Studies, 4, 155-161.

STROTZ, R. H. (1956). Myopia and inconsistency in dynamic utility maximization. Review of Economic Studies, 23, 165-180.

ThaleR, R. (1981). Some empirical evidence on dynamic inconsistency. Economics Letters, 8, 201-207.

\section{NOTE}

1. To test for possible differences in discounting rates, we fit the function

$$
V=1 /[1+(k+a * \Delta k) D],
$$

where $V$ is the subjective value of a delayed reward expressed as a proportion of its nominal amount, $k$ is the discount parameter for the larger amount, $\Delta k$ is the difference between the discount parameters for the larger and smaller amounts, and $a$ is an indicator variable that equals 0 for the larger amount and I for the smaller amount. To test for the significance of a difference in discounting rate, $\Delta k$ is divided by its estimated asymptotic standard deviation to obtain a value of $t$ with $n-p$ degrees of freedom, where $n$ is the number of data points (i.e., 14, or 7 per amount) and $p$ is the number of parameters (i.e., 2) in the model (Gallant, 1987).

(Manuscript received January 26, 1996 ; revision accepted for publication August 10, 1996.) 